\title{
Neuromuscular disorder as a presenting feature of coeliac disease
}

M Hadjivassiliou, A K Chattopadhyay, G A B Davies-Jones, A Gibson, R A Grünewald, A J Lobo

\begin{abstract}
Objectives-To describe the range of neuromuscular disorders which may be associated with cryptic coeliac disease.

Methods-Nine patients were described with neuromuscular disorders associated with circulating antigliadin antibodies, whose duodenal biopsies later confirmed the diagnosis of coeliac disease. Neurological symptoms antedated the diagnosis of coeliac disease in all, and most had minimal or no gastrointestinal symptoms at the onset of the neuromuscular disorder.

Results-Three patients had sensorimotor axonal peripheral neuropathy, one had axonal motor peripheral neuropathy, one had probable inclusion body myositis and axonal motor peripheral neuropathy, one had polymyositis and sensorimotor peripheral neuropathy, one had mononeuropathy multiplex, one had neuromyotonia, and one had polyneuropathy.

Conclusion-A wide range of neuromuscular disease may be the presenting feature of coeliac disease. This represents the first report of inclusion body myositis and neuromyotonia associated with coeliac disease. Estimation of circulating antigliadin antibodies should be considered in all patients with neuromuscular disease of otherwise obscure aetiology.
\end{abstract}

Department of

Neurology

$M$ Hadjivassiliou

G A B Davies-Jones

A Gibson

R A Grünewald

Department of Clinical Neurophysiology

A K Chattopadhyay

Department of Gastroenterology, Royal Hallamshire Hospital, Glossop

Road, Sheffield S10

2JF, UK

A J Lobo

Correspondence to: Dr M Hadjivassiliou, Department of Neurology, Royal Hallamshire Hospital, Glossop Road, Sheffield S10 2JF, UK.

Received 18 March 1997 and in revised form 17 June 1997 Accepted 30 June 1997
(F Neurol Neurosurg Psychiatry 1997;63:770-775)

Keywords: coeliac disease; gluten sensitivity; neuromuscular disorders

Neurological disorders associated with coeliac disease were first recognised at the turn of the century $^{1}$ and many case reports have been published since. Almost all of these papers report associations between established, often clinically severe, coeliac disease and neurological dysfunction. The range of neurological conditions associated with coeliac disease includes cerebellar ataxia, ${ }^{2}$ ataxia with myoclonus, ${ }^{3}$ peripheral neuropathy, ${ }^{4}$ myopathy, ${ }^{5}$ myelopathy, ${ }^{6}$ dementia, ${ }^{7}$ epilepsy, ${ }^{8}$ epilepsy with occipital calcifications, ${ }^{9}$ mononeuropathy multiplex, ${ }^{11}{ }^{11}$ progressive multifocal leukoencephalopathy, ${ }^{12}$ and vasculitis of the CNS. ${ }^{13}$

We have recently shown that asymptomatic coeliac disease is common in patients with diverse neurological conditions of unknown aetiology. ${ }^{10}$ In this paper we describe in detail the clinical presentation and electrophysiological findings of nine patients whose neuromuscular symptoms antedated the diagnosis of coeliac disease. Gastrointestinal symptoms were not prominent at presentation and in five of these patients they were absent, although one patient (case 3) had previously undergone resection for adenocarcinoma of the rectum which may have been a complication of untreated coeliac disease.

Most of these cases have been the subject of a brief presentation. ${ }^{14}$

\section{Patients}

Investigation of all nine patients disclosed the presence of antigliadin antibodies (IgA, IgG, or both). All patients underwent gastroscopy and distal duodenal biopsy which confirmed the diagnosis of coeliac disease. The median age at onset of neurological disease was 62 (range 17-76 years). The median delay from onset of neurological disorder to diagnosis of coeliac disease was one year (range six months to 13 years). All patients were extensively investigated for causes of neuromuscular disease and all other recognised causes excluded. With the exception of case 4 none had biochemical evidence of malabsorption (serum albumen, vitamin B12, folate, and iron were all normal).

CASE 1

A 62 year old woman had gradual onset over two years of stiffness of the ankles and a feeling of walking on "cotton wool". In addition she had experienced episodic transient numbness in the third and fourth fingers of the left hand. She had no relevant medical history but did recall episodic diarrhoea in childhood.

General physical examination was normal. Examination of the cranial nerves was unremarkable. In the limbs, muscular tone, power, and coordination were normal. Reflexes were normal and symmetric in the arms, but knee and ankle reflexes were absent and plantar responses flexor. There was a patchy sensory loss to light touch over both feet with preservation of vibration sense, pain perception, and joint position sense. The diagnosis of sensorimotor axonal peripheral neuropathy was confirmed electrophysiologically (table 1).

\section{CASE 2}

A 66 year old woman gradually developed weakness of the right leg and paraesthesia of the toes of the right foot over a six month period. Over the previous year she had experienced lower abdominal pain and urgency of 
defecation. She also described cramps in the buttocks and right leg made worse by exercise and eased by rest.

General examination was normal, as was examination of the cranial nerves and upper limbs. In the lower limbs tone was normal but there was weakness of ankle dorsiflexion and eversion bilaterally. Sensation in the left leg was normal. In the right leg there was loss of pain sensation, vibration sense, and light touch below the knee. A lumbar myelogram was normal; EMG showed distally predominant neuropathic changes.

By early 1991 weakness of the right leg had progressed and the patient was unable to walk more than five metres. She also developed increasing urinary frequency and urgency. MRI of the conus and lumbar spine was normal.

By 1994 the patient was dependent on a wheelchair. Electrophysiological examination showed an axonal sensorimotor peripheral neuropathy (table 1). At this time the diagnosis of coeliac disease was made.

CASE 3

A 67 year old man gradually noticed that he had to lift his legs high to avoid tripping . He also developed leg stiffness and a predominantly right sided rest tremor.

Thirteen years earlier he had undergone an anterioposterior resection for Duke's B rectal carcinoma. General examination disclosed a functioning colostomy but was otherwise normal. There was no evidence of tumour recurrence. He had a high stepping gait. There were fasciculations in the arms but no wasting. A resting tremor was present in the right hand. Muscle tone and power were normal except for moderate weakness of all ankle movements. Tendon reflexes were normal in the upper limbs but absent in the lower limbs. The plantar responses were flexor. There was loss of vibration sense below the knees and impairment of joint position sense in the toes bilaterally. Romberg's test was positive. Results of electrophysiological examination showed a sensorimotor axonal peripheral neuropathy (table 1).

Two years later coeliac disease was diagnosed although he still had no gastrointestinal symptoms.

CASE 4

A 76 year old man developed difficulty walking, weakness, and ascending sensory disturbance over one year. His complex medical history included pulmonary embolism, gastrointestinal haemorrhage, ischaemic heart disease, and chronic malabsorption ascribed to pancreatitis for which he took multivitamins, pancreatic enzyme supplements, and folic acid.

On examination there was mild gait ataxia. General physical examination and examination of the cranial nerves were normal. Tone and power in the limbs were normal apart from mild weakness of dorsiflexion of the ankles. He had absent tendon reflexes at the ankles and loss of joint position sense in the toes. Plantar responses were flexor.

Initial electrophysiological examination disclosed a distally predominant motor neuropathy with normal sensory nerve conduction studies (table 1). A repeat examination one year later showed progression to a sensorimotor axonal neuropathy.

The diagnosis of chronic pancreatitis was revised to that of coeliac disease after low duodenal biopsy.

\section{CASE 5}

A 49 year old woman developed painful weak muscles over a period of seven months, which were particularly noticeable when climbing stairs. Over a similar period she also experienced severe, episodic abdominal pain associated with diarrhoea. Medical history was unremarkable.

General examination was normal. Cranial nerves were intact. Tone in the limbs was normal, but there was moderate proximal weakness. Deep tendon reflexes were normal and

Table 1 Results of electrophysiological examination in cases 1-6

\begin{tabular}{|c|c|c|c|c|c|c|c|}
\hline \multirow[b]{4}{*}{ Case } & \multicolumn{6}{|c|}{ Nerve conduction studies } & \multirow{4}{*}{$\begin{array}{l}\text { Electromyography } \\
\text { Proximal and distal muscle in arm and leg }\end{array}$} \\
\hline & \multicolumn{4}{|l|}{ Sensory } & \multirow{2}{*}{\multicolumn{2}{|c|}{$\begin{array}{l}\text { Motor } \\
\text { Peroneal (EDB) }\end{array}$}} & \\
\hline & \multicolumn{2}{|c|}{ Median (digit II-wrist) } & \multicolumn{2}{|c|}{ Sural (lat mall-calf) } & & & \\
\hline & $S A P(\mu V)$ & $S C V(m / s)$ & $S A P(\mu V)$ & $S C V(m / s)$ & $C M A P(m V)$ & $M C V(\mathrm{~m} / \mathrm{s})$ & \\
\hline 1 & 4.4 & 57 & 4.4 & 49 & 0.8 & 49 & $\begin{array}{l}\text { Distally predominant neuropathic changes (long duration, } \\
\text { polyphasic MUP, and reduced IP) }\end{array}$ \\
\hline 2 & 6.3 & 55 & 2.3 & 34 & 0 & - & $\begin{array}{l}\text { Distally predominant neuropathic changes (positive sharp } \\
\text { waves in VM and TA, long MUP, and reduced IP in VM, no } \\
\text { MUP in TA) }\end{array}$ \\
\hline 3 & 3.7 & 47 & 1.5 & 38 & 0 & - & $\begin{array}{l}\text { Distally predominant neuropathic changes (no MUP in EDB, } \\
\text { long duration MUP in others) }\end{array}$ \\
\hline 4 & 12 & 54 & 5.8 & 36 & 1.9 & 49 & $\begin{array}{l}\text { Distally predominant neuropathic changes (long duration } \\
\text { MUP and reduced IP) }\end{array}$ \\
\hline 5 & 13 & 50 & 4 & 48 & - & 47 & $\begin{array}{l}\text { Myopathic and distally predominant neuropathic changes } \\
\text { (short and long duration MUP, reduced IP) }\end{array}$ \\
\hline 6 & - & - & 5.3 & 43 & 0.1 & 36 & $\begin{array}{l}\text { Myopathic and distally predominant neuropathic changes } \\
\text { (short polyphasic and long duration MUP, reduced IP) }\end{array}$ \\
\hline
\end{tabular}

SAP $=$ sensory action potential amplitude (normal $>5 \mu \mathrm{V}) ; \mathrm{SCV}=$ sensory nerve conduction velocity (normal $>48 \mathrm{~m} / \mathrm{s}$ in arm and $>38 \mathrm{~m} / \mathrm{s}$ in leg); Lat mall $=$ lateral malleolus; $\mathrm{EDB}=$ extensor digitorum brevis; $\mathrm{CMAP}=$ compound muscle action potential (normal $>1 \mathrm{mV}$ recording from $\mathrm{EDB}$ with surface electrodes and stimulating peroneal nerve at the front of the ankle); MCV = motor nerve conduction velocity (normal $>48 \mathrm{~m} / \mathrm{s}$ in arm and $>38 \mathrm{~m} / \mathrm{s}$ in leg) - denotes not examined; $\mathrm{MUP}=$ motor unit potentials; $\mathrm{IP}=$ interference patterns; $\mathrm{VM}=$ vastus medialis; $\mathrm{TA}=$ tibialis anterior. 
plantar responses flexor. Coordination and sensation were also intact.

Her serum creatine phosphokinase concentration was $>6500 \mathrm{u} / 1$ (normal range 25-190 $\mathrm{u} / 1$ ). A muscle biopsy was performed which showed features characteristic of polymyositis. Electrophysiological examination showed both a myopathy and a sensorimotor axonal peripheral neuropathy (table 1 ).

The patient was treated with prednisolone with a good clinical response. She was discharged from the clinic in 1984.

Ten years later she was rereferred to the neurology service by a gastroenterologist who had diagnosed coeliac disease some two years earlier. One year after the diagnosis of coeliac disease she developed an unsteady gait and paraesthesia of her hands and feet. She also complained of a burning sensation of both feet. Although she had been on a gluten free diet at that time her antigliadin antibody titres were positive.

On examination there was pronounced gait ataxia. There was wasting and weakness of the intrinsic hand muscles bilaterally with hyporeflexia in the arms. There was moderately severe weakness of ankle dorsiflexion bilaterally and areflexia in the legs with distally reduced discrimination of pain, light touch, and vibration sense. Coordination was poor in her upper and lower limbs. Plantar responses were flexor.

Creatine phosphokinase was $621 \mu \mathrm{m} / 1$. Further electrophysiological examination showed deterioration of the peripheral neuropathy. Myopathic features were still present. Steroid treatment was recommenced.

CASE 6

A 64 year old man developed progressive weakness in the legs, more pronounced proximally, resulting in several falls. $\mathrm{He}$ also complained of episodic swallowing difficulties.
Six months after the onset of his weakness he was investigated for diarrhoea and weight loss and a diagnosis of coeliac disease was made.

$\mathrm{He}$ was a thin man with bilateral $\mathrm{Du}-$ puytren's contractures. There was pronounced bulbar weakness and dysphagia. Tone in the limbs was normal, but there was a moderate proximal weakness. Tendon reflexes were generally depressed. Joint position sense was absent in the toes and vibration sense was absent in the ankles. Perception of light touch and pain were normal.

His serum creatine phosphokinase was raised to $650 \mathrm{u} / 1$. Muscle biopsy showed typical features of an inflammatory myopathy. Many fibres within the biopsy showed basophilic rimmed vacuoles suggestive of inclusion body myositis (fig 1). Electrophysiological examination suggested both a myopathy and a motor axonal peripheral neuropathy (table 1). Repeat examination four years later showed progression to a sensorimotor neuropathy.

\section{CASE 7}

A 45 year old man developed numbness of the anterior aspect of his right lower leg and dorsum of the right foot. He also gave a history of weakness of the right leg with occasional stumbling to the right hand side.

Results from a general examination and examination of the cranial nerves were normal. In the limbs tone and power were normal. There was loss of vibration and joint position sense in both legs and loss of pain perception in the whole of the right leg to the groin. Tendon reflexes in the arms were normal but were absent in the lower limbs. The initial nerve conduction study was normal and EMG showed distal motor neuropathic changes. A lumbar myelogram was entirely normal.

His condition deteriorated and within two years he had developed patchy numbness in the

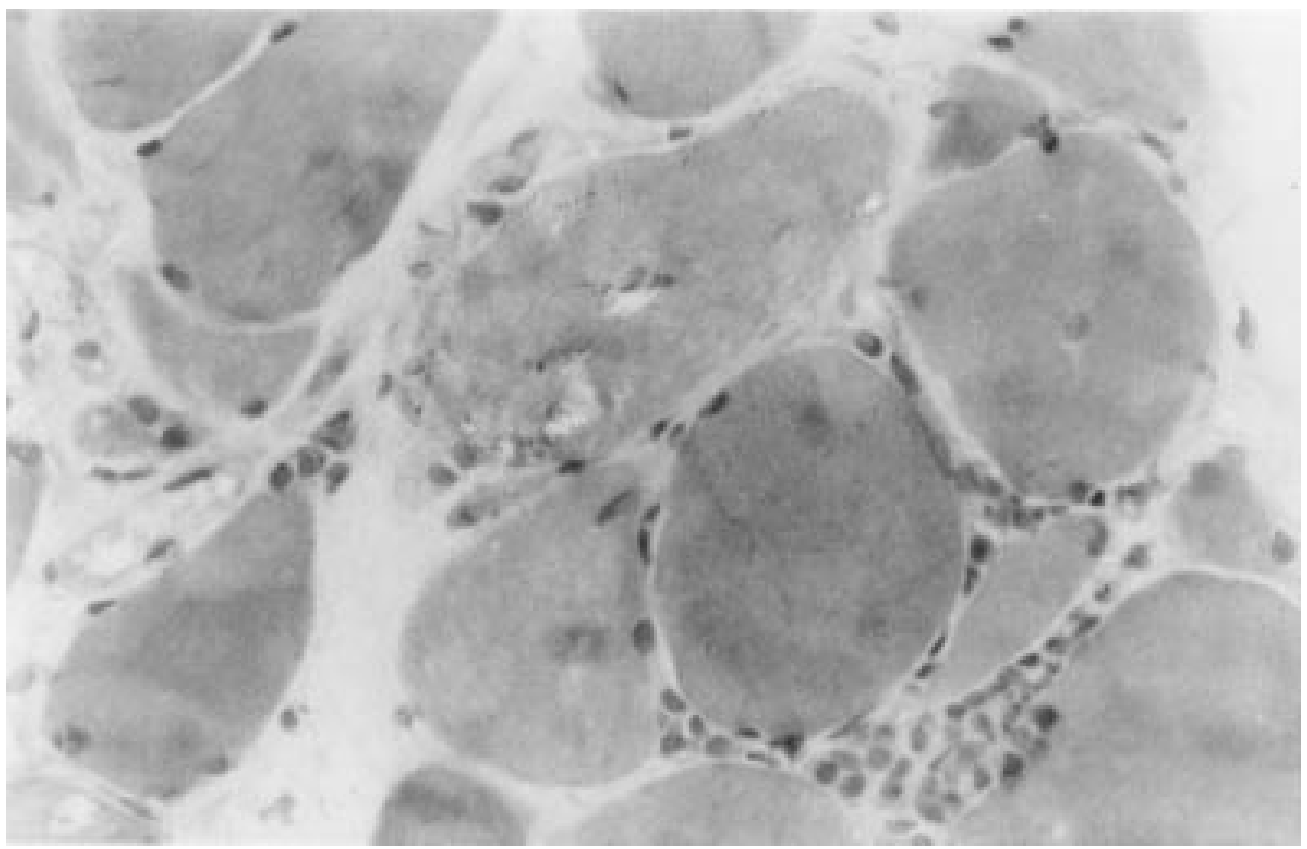

Figure 1 Muscle biopsy from case 6, showing inflammatory infiltration, variation in fibre size, and basophilic rimmed vacuoles characteristic of inclusion body myositis. 
Table 2 Results of electrophysiological examination in case 7

\begin{tabular}{|c|c|c|c|c|c|c|}
\hline \multirow[b]{2}{*}{ Sensory } & \multicolumn{2}{|c|}{ 1st examination } & \multicolumn{2}{|c|}{ 2nd examination } & \multicolumn{2}{|c|}{ 3rd examination } \\
\hline & $S A P(\mu V)$ & $S C V(m / s)$ & $S A P(\mu V)$ & $S C V(m / s)$ & $S A P(\mu V)$ & $S C V(m / s)$ \\
\hline \multicolumn{7}{|c|}{ Median (digit II-wrist) } \\
\hline Right & 3.8 & 65 & 1.8 & 67 & 2.7 & 61 \\
\hline Left & - & - & - & - & 2.6 & 58 \\
\hline \multicolumn{7}{|c|}{ Ulnar (digit V-wrist) } \\
\hline Right & 0 & & 0 & & 0 & \\
\hline Left & - & - & - & - & 3.3 & 50 \\
\hline \multicolumn{7}{|c|}{$\begin{array}{l}\text { Radial (forearm-lst dorsal } \\
\text { interosseous space) }\end{array}$} \\
\hline Right & 27 & 51 & 12 & 61 & 7.2 & 58 \\
\hline Left & - & - & - & - & 0 & \\
\hline \multicolumn{7}{|c|}{ Sural (lateral malleolus-calf) } \\
\hline Right & 7.1 & 4.6 & 2.2 & 44 & 1.6 & 44 \\
\hline Left & - & - & - & - & 7.2 & 31 \\
\hline
\end{tabular}

Motor nerve conduction studies in the right median, right ulnar and right peroneal nerves were normal in all three examinations. $\mathrm{SAP}=$ sensory action potential amplitude $($ normal $>5 \mu \mathrm{V})$; SCV = sensory nerve conduction velocity (normal $>48 \mathrm{~m} / \mathrm{s}$ in arm and $>38 \mathrm{~m} / \mathrm{s}$ in leg); - denotes not examined.

right arm with loss of the right triceps reflex. A clinical and electrophysiological diagnosis of mononeuritis multiplex was made (table 2, first examination), but investigation disclosed no aetiology. He returned in 1993 with a gradual onset of weakness in the intrinsic muscles of both hands. He also complained of intermittent lower abdominal pain. The diagnosis of coeliac disease was made in 1994. Two further electrophysiological examinations have been made since (table 2, second and third examinations).

CASE 8

An 18 year old student developed progressively worsening general fatigue, cramps, and stiffness in the hands, arms, and, to a lesser extent, the legs. The stiffness in his hands and arms was severe enough to stop him playing basketball but did not interfere with his ability to run. He had also noticed a continuous rippling in

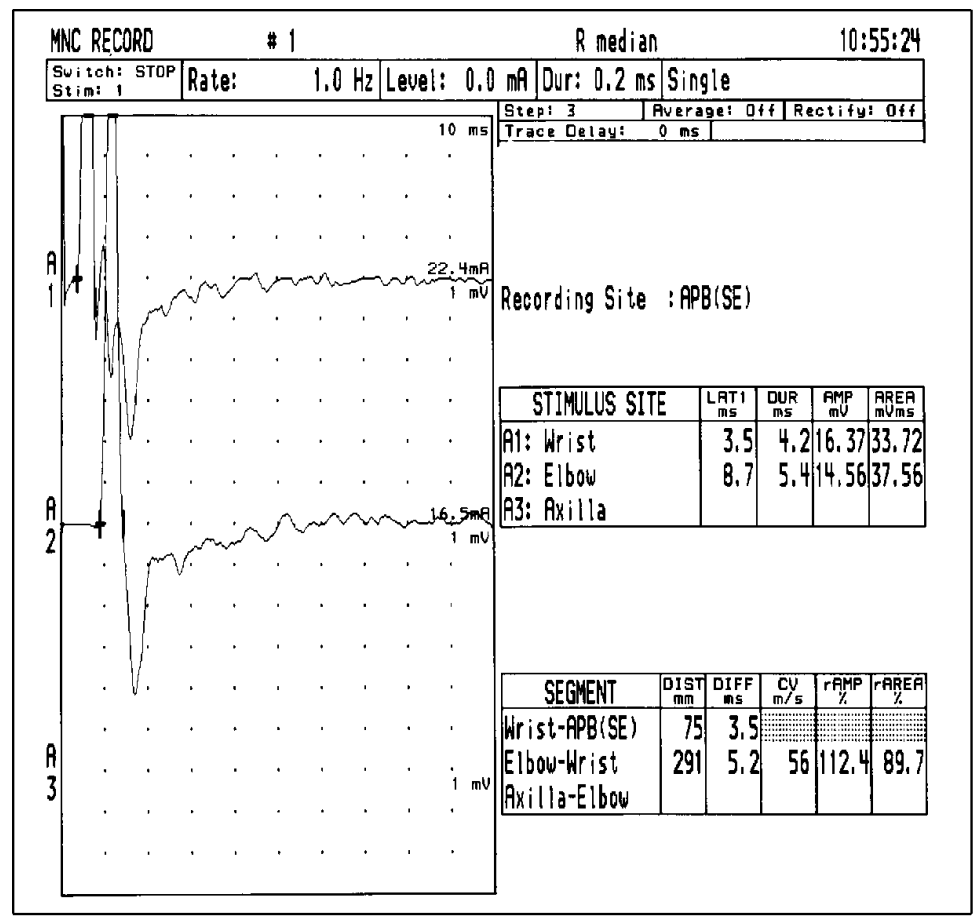

Figure 2 Median motor nerve conduction study showing afterdischarges lasting for about 80 ms from the $M$ wave (case 8). Time base is 10 ms per division. the muscles of his arms and calves and a modest reduction in grip strength.

The condition had followed an illness characterised by a sore throat (treated with antibiotics) and a severe maculopapular rash. $\mathrm{He}$ denied any gastrointestinal symptoms.

Examination disclosed increased tone in the arms, the left more than the right. Power in the arms and legs was normal but there was delay of relaxation after voluntary contraction. There was widespread myokymia of the face, arms, shoulder girdle, and lower limbs. The reflexes were absent in the upper limbs but normal in the legs.

Antipotassium channel antibodies and antiglutamic acid decarboxylase antibodies were normal. Electrophysiological examination showed normal sensory and motor conduction velocities. Repetitive motor nerve stimulation consistently showed afterdischarges (fig 2). Electromyography showed fasciculations and neuromyotonic discharges. The findings were those of acquired neuromyotonia. The diagnosis of coeliac disease was made six months later.

\section{CASE 9}

A 52 year old woman developed an erythematous rash in a butterfly distribution on her face followed by generalised weakness. Two days later she developed paraesthesia of both feet spreading to the arms. Within 48 hours she was unable to walk due to profound weakness and developed respiratory difficulties. Results of CSF examination were normal.

On examination she was found to have reduced tone with weakness affecting both arms and legs. She was areflexic and had mild reduction of sensory modalities distally. Her forced vital capacity was reduced to 1.2 litres. A clinical diagnosis of Guillian-Barré syndrome was made.

She was treated with intravenous immunoglobulins and showed pronounced improvement within a week of treatment. No neurophysiological examination was performed at this stage of her illness. Investigations showed her to have circulating antigliadin antibodies, but the relevance was uncertain in view of previous treatment with intravenous immunoglobulin. She was transferred to the district general hospital for a period of rehabilitation. 
During this period she developed diarrhoea associated with abdominal cramps. Stool cultures showed no evidence of an infective cause. Serum antinuclear antibodies were absent. After discharge she continued to complain of diarrhoea as well as persistence of paraesthesia and generalised weakness. Distal duodenal biopsy confirmed the presence of coeliac disease. Nerve conduction studies at this stage showed that the amplitude of the compound muscle action potential recorded from the extensor digitorum brevis on stimulation of the peroneal nerve was at the lower limit of normal $(1 \mathrm{mV})$. Few, and mostly delayed, $\mathrm{F}$ waves were recorded on stimulating the median nerve and none on stimulating the peroneal nerve, consistent with a resolving neuropathy with a demyelinating component.

\section{Discussion}

Neurological disorders associated with overt coeliac disease have been the subject of more than 60 articles with considerable heterogeneity of neurological features. We think that this is the first description of an association of coeliac disease with neuromyotonia and inclusion body myositis.

Although neurological disorders have been reported in association with established and often severe coeliac disease, neurological disorders antedating symptoms and diagnosis of coeliac disease have as yet only been reported by our group. We have previously reported a high prevalence of gluten sensitivity in patients with neurological dysfunction of unknown cause, which implies that this association with neurological disease is not merely chance. ${ }^{10}$ The cases we describe here represent the range of neuromuscular disorders we have found in association with cryptic gluten sensitivity, and illustrate that coeliac disease must be considered in the diagnosis of neuromuscular disorders of otherwise obscure aetiology.

The variety of neuromuscular dysfunction associated with cryptic coeliac disease is wide and often progressive. Two patients (cases 4 and 6) initially presented with motor axonal peripheral neuropathy. Subsequent examination showed sensory abnormalities on electrophysiological examination, implying that gluten sensitivity should be considered in patients presenting with a cryptogenic pure motor neuropathy. The influence of a gluten free diet on the progression of these neuromuscular disorders remains unknown.

Nutritional deficiency is an unlikely explanation for neuromuscular disorders in these patients for the following reasons: firstly, many of the neuromuscular disorders described in this and other reports clearly have an inflammatory or autoimmune basis, including polymyositis, mononeuropathy multiplex, acute polyneuropathy, and neuromyotonia. Secondly, the neurological disorders in our patients preceded the gastrointestinal disorder by many years, and the patients had no physical or biochemical evidence of malabsorption at the onset of neurological disease. Thirdly, we have described neurological disorders associated with gluten sensitivity but normal small bowel mucosa, ${ }^{10}$ making appreciable malabsorption unlikely.

An immunological role may be important in the genesis of the neurological dysfunction associated with coeliac disease. ${ }^{10}$ Advances in the understanding of coeliac disease have necessitated modification of the definition of the condition based solely on small bowel histology. Distal duodenal biopsies in patients with gluten sensitivity disclose a range of mucosal damage extending from an infiltrative to a hypoplastic atrophic picture. ${ }^{15}$ Rather than being defined by small bowel histology, coeliac disease is now defined as an abnormal immunological response to ingested gliadin in genetically susceptible people. ${ }^{16}$

The concept of "latent" coeliac disease has also emerged. ${ }^{15}{ }^{16}{ }^{17} \mathrm{~A}$ reversal in the ratio of $\alpha / \beta$ to $\gamma / \delta$ subpopulations of intraepithelial $\mathrm{T}$ lymphocytes in the small bowel mucosa has been found to be a marker of latent coeliac disease. ${ }^{18}$ The role of $\gamma / \delta \mathrm{T}$ cells remains elusive but their implication in inflammatory neurological disease has been the subject of extensive research. Cultured $\mathrm{T}$ lymphocytes from a nerve biopsy from a patient with acute demyelinating polyneuropathy were shown to be of the $\gamma / \delta$ subpopulation. ${ }^{19}$

The vast majority of patients with coeliac disease carry one particular HLA variant, DQ2. The few remaining patients seem in most cases to carry HLA DQ8. Exposure of lamina propria $\mathrm{T}$ cells to gliadin in these patients results in a broad cytokine secretion pattern (interleukins, interferon, tumour necrosis factor). This results in proliferation of $\mathrm{DQB}_{1} 0201$ restricted intraepithelial $T$ cell clones in the small bowel mucosa which may circulate. Peripheral blood $\mathrm{T}$ cells from most patients with coeliac disease respond strongly to gliadin in vitro. ${ }^{20}$ Circulating activated $\mathrm{T}$ cells may initiate an immune response in tissues other than the small bowel.

The role of the humoral responses is uncertain. The number of IgA secreting plasma cells within the mucosa of the small bowel is significantly increased, but gluten sensitivity is considerably commoner in subjects with $\operatorname{IgA}$ deficiency. High concentrations of antigliadin antibodies are found systemically, but it is not yet known if these antibodies are potentially neurotoxic.

Our finding that neuromuscular disorders can be the presenting feature of coeliac disease implies that the immune response triggered by sensitivity to gluten may find expression in organs other than the gut and that the central and peripheral nervous systems are particularly susceptible. The skin is another target organ as in the case of patients with dermatitis herpetiformis $(95 \%$ of these patients have "mild" coeliac disease).

The abnormal immunological response triggered by ingested gluten in genetically susceptible people can be arrested by strict adherence to a gluten free diet. Even minute traces of gliadin are capable of triggering a state of heightened immunological activity in gluten sensitive people. Strict adherence to gluten free 
diet is therefore essential but cannot be monitored by the presence of gastrointestinal symptoms as most of our patients had none. Quantitative antigliadin antibody assays may be a useful monitoring tool, and are being employed in our current evaluation of the impact of a gluten free diet on neurological dysfunction associated with gluten sensitivity.

We thank the following people who contributed towards this Royal Hallamshire Hospital, Dr R H Kandler, consultant clinical neurophysiologist, Royal Hallamshire Hospital, Dr P Moorhead, consultant physician, Northern General Hospital, Dr C M L Smith, consultant neuropathologist, Royal Hallamshire Hospital.

1 Brown WC. Sprue and its treatment. London: Bale, 1908, cited in Marsh. ${ }^{16}$

2 Elders C. Tropical sprue and pernicious anaemia, aetiology and treatment. Lancet 1925;i:75-7.

3 Lu CS, Thompson PD, Quin NP, Parkes JD, Marsden CD Ramsay Hunt syndrome and coeliac disease: a new association. Mov Disord 1986;1:209-19.

4 Cooke WT, Thomas-Smith W. Neurological disorders associated with adult coeliac disease. Brain 1966;89:683-722

5 Henriksson KG, Hallert C, Norrby K, Walan A. PolymyosiHenriksson KG, Hallert C, Norrby K, Walan A. Polymyosi-
tis and adult coeliac disease. Acta Neurol Scand 1982;65: tis and adu.

6 Sencer W. Neurologic manifestations in the malabsorption syndrome. Fournal of the Mount Sinai Hospital 1957;24 331-45.

7 Collin P, Pirttila T, Nurmikko T, Somer H, Erila T, Keyrilainen O. Celiac disease, brain atrophy, and dementia. Neurology 1991;41:372-5.

8 Chapman RWG, Laidlow JM, Colin-Jones D, Eade OE, Smith CL. Increased prevalence of epilepsy in coeliac disease. $B M \mathcal{F}$ 1978;ii:250-1.
9 Gobbi G, Bouquet F, Greco L, Lambertini A, Tassinari CA, Ventura A, Zaniboni MG. Coeliac disease, epilepsy, and cerebral calcifications. Lancet 1992;340:439-43.

10 Hadjivassiliou M, Gibson A, Davies-Jones GAB, Lobo A, Stephenson TJ, Milford-Ward A. Is cryptic gluten sensitivity an important cause of neurological illness? Lancet 1996; 347:369-71.

11 Kelkar P, Ross MA, Murray J. Mononeuropathy multiplex associated with celiac sprue. Muscle Nerve 1996;19:234-6.

12 Kepes JJ, Chou SM, Price LW. Progressive multifocal leukoencephalopathy with 10 -year survival in a patient with non-tropical sprue. Neurology 1975;25:1006-12.

13 Rush PJ, Inman R, Bernstein M, Carlen P, Resch L. Isolated vasculitis of the central nervous system in a pateint with coeliac disease. Am F Med 1986;81:1092-4.

14 Gibson A, Hadjivassiliou M, Chattopadhyay AK, DaviesJones GAB. Cryptic coeliac disease presenting with neuromuscular dysfunction. F Neurol Neurosurg Psychiatry 1997;62:204.

15 Marsh MN. Gluten major histocompatibility complex, and the small intestine: a molecular and immunobiologic approach to the spectrum of gluten sensitivity. Gastroenterology 1992;102:330.

16 Marsh MN. The natural history of gluten sensitivity: defining, refining and re-defining. $Q \mathcal{F}$ Med 1995;85:9-13.

17 Maki M, Holm K, Collin P, Savilahti E. Increased in gamma/delta T-cell receptor bearing lymphocytes in gamma/delta T-cell receptor bearing lymphocytes in 1991;32:1412-4.

18 Halstensen TS, Farstad IN, Scott H. Intraepithelial T cells of the TCR gamma/delta CD8 and Vdelta 1/Jdelta 1 phenotypes are increased in coeliac disease. Scand 7 Immunol 1989;39:665.

19 Smith AB, Gaston JSH, Barber PC, Winer JB. Isolation of T-lymphocytes from nerve biopsy in Guillian-Barre syndrome. F Neurol Neurosurg Psychiatry 1996;61:215.

20 Gjertsen HA, Sollid LM, Ek J, Thorsby E, Lundin KEA. T cells from the peripheral blood of coeliac disease patients recognise gluten antigens when presented by HLA-DR, -DQ or -DP molecules. Scand f Immunol 1994;39:567-74. 